Journal of Computer Science 6 (10): 1199-1202, 2010

ISSN 1549-3636

(C) 2010 Science Publications

\title{
Neural Network Based Accurate Biometric Recognition and Identification of Human Iris Patterns
}

\author{
${ }^{1}$ M. Gopikrishnan and ${ }^{2}$ T. Santhanam \\ ${ }^{1}$ Prathyusha Institute of Technology and Management, Chennai-602 025, India \\ ${ }^{2}$ Department of Computer Science, Reader and Head PG and Research, \\ DG Vaishmav College, Chennai-600 106, India
}

\begin{abstract}
Problem statement: A biometric system provides automatic identification of an individual based on a unique feature or characteristic possessed by the individual. Iris recognition is regarded as the most reliable and accurate biometric identification system available. Approach: Most commercial iris recognition systems use patented algorithms developed by Daugman and these algorithms are able to produce perfect recognition rates. However, published results have usually been produced under favorable conditions and there have been no independent trials of the technology. Results: In this study after providing brief picture on development of various techniques for iris recognition, hamming distance coupled with neural network based iris recognition techniques were discussed. Perfect recognition on a set of 150 eye images has been achieved through this approach. Further, Tests on another set of 801 images resulted in false accept and false reject rates of 0.0005 and $0.187 \%$ respectively, providing the reliability and accuracy of the biometric technology. Conclusion/Recommendations: This study provided results of iris recognition performed applying Hamming distance, Feed forward back propagation, Cascade forward back propagation, Elman forward back propagation and perceptron. It has been established that the method suggested applying perceptron provides the best accuracy in respect of iris recognition with no major additional computational complexity.
\end{abstract}

Key words: Iris recognition, biometric identification, pattern recognition, automatic segmentation

\section{INTRODUCTION}

The iris is the colored portion of the eye that surrounds the pupil. It is full of richly textured patterns that are distinct from person to person and in fact are distinct from left eye to right eye from the same person. Compared with other biometric features such as face and fingerprint, iris patterns are more stable and reliable and are unrelated to health or the environment (Woodward et al., 2002). Iris recognition systems are noninvasive to their users, but do require a cooperative subject. For this reason, iris recognition is usually used for verification or identification purposes.

The key step in most current iris pattern recognition algorithms is to convert the iris pattern into a two dimensional code (Daugman, 1993; Wildes et al., 1996; Boles and Boashash, 1998). To eliminate the effect of eye tilt, circular rotation of the iris pattern is usually necessary in iris matching and identification algorithms. Among them, Daugman's 2D Gabor wavelet approach has been successfully tested using a large-scale iris database and has been commercialized by Iridian (Siedlarz, 1994).

In this study after providing brief picture on development of Various techniques for iris recognition, Hamming distance coupled with Neural Network based iris recognition techniques are discussed. Perfect recognition on a set of 150 eye images has been achieved through this approach. Further, Tests on another set of 801 images resulted in false accept and false reject rates of $0.0005 \%$ and $0.187 \%$ respectively, providing the reliability and accuracy of the biometric technology. This study provides results of iris recognition performed applying Hamming distance, Feed forward back propagation, Cascade forward back propagation, Elman forward back propagation and perceptron. It has been established that the method suggested applying perceptron provides the best accuracy in respect of iris recognition with no major additional computational complexity. In this study use the CASIA iris image database collected by Institute of Automation, Chinese Academy of Sciences (CASIA, 2008). 


\section{MATERIALS AND METHODS}

Image preprocessing: A sample iris image is shown in Fig. 1. Since it has a circular shape when the iris is orthogonal to the sensor, iris recognition algorithms typically convert the pixels of the iris to polar coordinates for further processing. An important part of this type of algorithm is to determine which pixels are actually on the iris, effectively removing those pixels that represent the pupil, eyelids and eyelashes, as well as those pixels that are the result of reflections.

In this algorithm, the locations of the pupil and upper and lower eyelids are determined first using edge detection. This is performed after the original iris image has been down sampled by a factor of two in each direction (to $1 / 4$ size, in order to speed processing). The best edge results came using the canny method (Canny, 1986). An example is shown in Fig. 2, where the top is the original iris image and the bottom is the edge detection results. The pupil clearly stands out as a circle and the upper and lower eyelid areas above and below the pupil are also prominent. A Hough transform is then used to find the center of the pupil and its radius.

Once the center of the pupil is found, the original image is transformed into resolution invariant polar coordinates using the center of the pupil as the origin. This is done since the pupil is close to circular. Although not always correct, it is assumed that the outer edge of the iris is circular as well, also centered at the center of the pupil. From this geometry, when the original image is transformed into polar coordinates, the outer boundary of the iris will appear as a straight (or near straight) horizontal line segment (Fig. 3 (top)). This edge is determined using a horizontal sober filter.

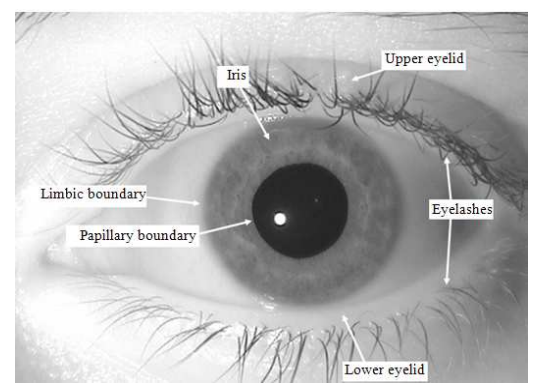

Fig. 1: Sample near infrared iris image
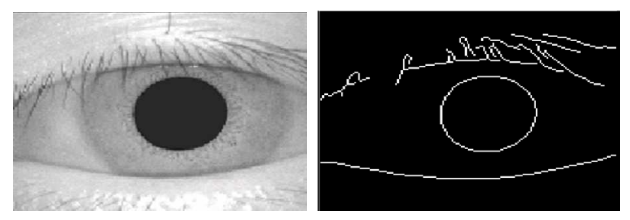

Fig. 2: Iris image and its edges using canny method
After determination of the inner and outer boundaries of the iris, the non-iris pixels within these concentric circles must be determined (Fig. 3 (bottom)). Thresholding identifies the glare from reflections (bright spots), while edge detection issued to identify eyelashes.

Enrollment: Enrollment is the process of generating some representation of the iris that is to be stored in the database for use in identification. Typically, this involves combining several images of the same iris in some manner in order to produce a representative sample that has less noise than any individual image. For this system, the normalized iris images and the result stored in the database as the template are compared for identification. The enrollment process includes image acquisition, image preprocessing and the template creation.

Identification: The identification process, in which a new iris image, presented to the system, undergoes the same preprocessing like that of the iris images in the enrollment database. The normalized template is then compared to each template in the enrollment database to determine a match.

Neural network distance: Artificial neural networks have been explored for iris identification. Many approaches, such as formulating the identification problem such that the neural network would identify the individual irises (i.e., the neural network contains an output class for each individual iris). This means a neural network that could identify 100 people would have 200 output nodes, each representing an individual iris. This architecture is typically useful for small databases, but requires enlarging and retraining of the neural network as the database grows.
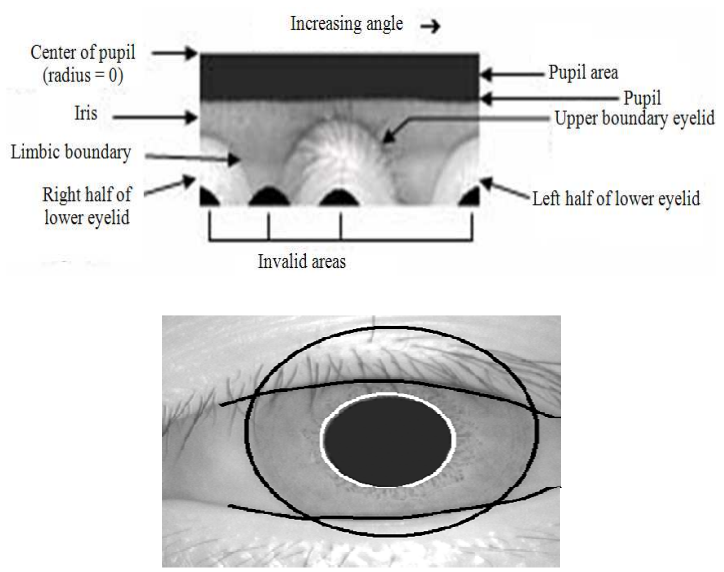

Fig. 3: Iris image in polar coordinates (top) and original image after inner and outer edges of iris determined (bottom) 
Table 1: Comparison between experimental results

\begin{tabular}{lllllll}
\hline $\begin{array}{l}\text { Neural } \\
\text { Network tools }\end{array}$ & $\begin{array}{l}\text { Exp. result } \\
\text { analysis 1 }\end{array}$ & $\begin{array}{l}\text { Exp. result } \\
\text { analysis 2 }\end{array}$ & $\begin{array}{l}\text { Exp. result } \\
\text { analysis 3 }\end{array}$ & $\begin{array}{l}\text { Exp. result } \\
\text { analysis 4 }\end{array}$ & $\begin{array}{l}\text { Exp. result } \\
\text { analysis 5 }\end{array}$ & $\begin{array}{l}\text { Exp. result } \\
\text { analysis 6 }\end{array}$ \\
\hline Hammington distance & 0.3418 & 0.2509 & 0.2822 & 0.2427 & 0.2480 & 0.4154 \\
Feed forward back propagation & 0.0740 & 0.0562 & 0.1083 & 0.1590 & 0.0401 & 0.0522 \\
Cascade forward back propagation & 0.1592 & 0.0777 & 0.2091 & 0.1060 & 0.1761 & 0.2082 \\
Elman forward back propagation & 0.0740 & 0.0562 & 0.0569 & 0.9013 & 0.0403 & 0.0512 \\
Perceptron & 0.0083 & 0.0045 & 0.0056 & 0.0085 & 0.0083 & 0.0022 \\
\hline
\end{tabular}

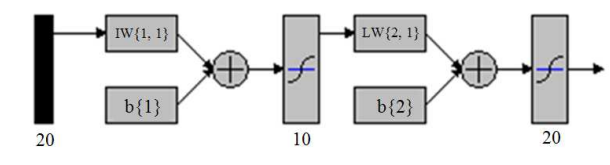

Fig. 4: Feed-forward back propagation

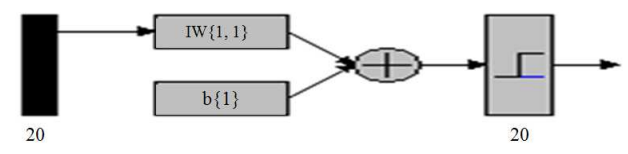

Fig. 5: Perceptron

In this study, a neural network is used to identify the statistical pattern present when one iris template matches or does not match another. The neural network can be small (thus fast) and will contain only two output nodes representing a match or a non-match. The neural network need not be retrained as individuals are added to the database. A Feed Forward Back Propagation (FFBP) and perceptron artificial neural network, shown in Fig. 4 and 5 has been used to form the match decision. The error back-propagation training algorithm is used to adjust the internal neural network weights.

\section{RESULTS ADN DISCUSSION}

The performance of the proposed neural network tool is evaluated with CASIA database (the institute of Automation, Chinese Academy of Sciences). The CASIA data base contain nearly 4500 iris images at $(320 \times 280)$. The experiments were carried out in Intel core 2 Duo processor with $1.83 \mathrm{GHz}$ with $1 \mathrm{~GB}$ DDR 2 for all iris sample templates. This is a real world application level simulation. The experimentation is conducted in two stages:

- Performance evaluation of the proposed neural network tool approach

- Comparison with the existing approaches in the field of iris recognition. In the first stage of the experimentation. Emphasis is on the performance evaluation of the current approach based on the matching accuracy. Evaluation of the proposed method is by comparing its recognition accuracy with the matching strategies. The performance of the proposed genetic process is demonstrated in Table 1 through selection of optimum features as well as increase in the overall system accuracy.

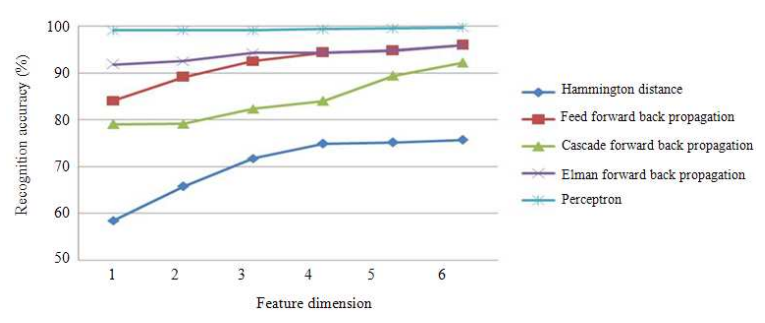

Fig. 6: Verification of performance using ROC curves

The verification performance of the proposed approach is shown in Fig. 6 using a Receiver Operator Characteristics (ROCs) curve. The effect of performance evaluation on different security requirements is obtained by changing the values of weight ages. The experimental results measure the probability of accepting an imposter as an authorized subject and the probability of rejecting an authorized subject incorrectly. During the second stage, through a series of experimentation, a comparative analysis of the suggested method with the existing methods, in respect of recognition accuracy and computational complexity, is provided

\section{CONCLUSION}

In this study, a new iris recognition method is proposed using an efficient iris segmentation approach, based on the collarette area localization, with the incorporation of the eyelashes and the eyelids detection techniques. The 1D log-Gabor filters are used to extract the discriminating features. In order to increase the matching accuracy neural network tools have been applied. Experimental results exhibit an encouraging performance as for as the accuracy is concerned especially on the CASIA data set. A comparative analysis among the existing schemes of the iris recognition has been conducted. The performance evaluation and comparisons with other methods indicate that the proposed method is a viable and very efficient method for iris recognition. In future, the Counter propagation and SVM tool will be used to conduct the experiments to improve the accuracy of overall matching iris recognition system. 


\section{ACKNOWLEDGMENT}

The iris CASIA dataset is available on the web at http://www.sinobiometrics.com/english/Iris\%20Databas es.asp (CASIA, 2008).

\section{REFERENCES}

Boles, W.W. and B. Boashash, 1998. A human identification technique using images of the iris and wavelet transform. IEEE Trans. Sign. Process., 46: 1185-1188.

Canny, J., 1986. A computational approach to edge detection. IEEE Trans. Patt. Anal. Mach., 8: 679-698.

CASIA, 2008. Iris image database. Sinobiometrics. http://www.sinobiometrics.com
Daugman, J., 1993. High confidence visual recognition of persons by a test of statistical independence. IEEE Trans. Patt. Anal. Mach. Intell., 15: 1148-1161.

Siedlarz, J.E., 1994. Iris: More detailed than a fingerprint. IEEE Spectrum, 31: 27-27.

Wildes, R.P., J.C. Asmuth, G.L. Green, S.C. Hsu and R.J. Kolczynski et al., 1996. A machine vision system for iris recognition. Mach. Vis. Appli., 9: 1-8.

Woodward, Jr., J.D., N.M. Orlans and P.T. Higgins, 2002. Biometrics: Assurance in the Information Age. 1st Edn., The McGraw-Hill Company, Berkeley, California, ISBN: 10: 0072222271, pp: 416. 